PHYSICAL REVIEW B 85, 174428 (2012)

\title{
Interaction between propagating spin waves and domain walls on a ferromagnetic nanowire
}

\author{
J.-S. Kim, M. Stärk, and M. Kläui* \\ Fachbereich Physik, Universität Konstanz, Universitätsstr. 10, D-78457 Konstanz, Germany \\ J. Yoon and C.-Y. You ${ }^{\dagger}$ \\ Department of Physics, Inha University, Incheon 402-751, Republic of Korea \\ L. Lopez-Diaz and E. Martinez \\ Universidad de Salamanca, Plaza de la Merced s/n, E-37008, Salamanca, Spain \\ (Received 15 July 2011; revised manuscript received 14 February 2012; published 21 May 2012)
}

\begin{abstract}
We numerically investigate the interaction between propagating spin waves and a transverse domain wall in a nanowire by using micromagnetic simulations. In order to understand the mechanisms that lead to domain wall motions, we calculate domain wall velocity in a defect-free nanowire and the depinning fields for a pinned domain wall that is depinned in and against the direction of the spin-wave propagation. We find that the physical origin of the spin-wave-induced domain wall motion strongly depends on the propagating spin-wave frequency. At certain spin-wave frequencies, transverse domain wall vibrations lead to transverse wall displacements by the spin waves, while at other frequencies, large spin-wave reflection drives domain wall motion. By analyzing the depinning field calculations, the different underlying physical mechanisms are distinguished.
\end{abstract}

DOI: 10.1103/PhysRevB.85.174428

PACS number(s): 75.30.Ds, 75.60.Ch

\section{INTRODUCTION}

Different approaches have been suggested to induce domain wall (DW) displacements as required for devices based on DW motion. ${ }^{1-3}$ The traditional approaches using external fields faces scaling problems, because as the size of the device is reduced, the current density needed to generate a sufficiently large Oersted field to depin the DW increases. ${ }^{4}$ On the other hand, current-induced DW motion via the spin torque effect has attracted significant attention lately because it opens an alternative path to induce efficient magnetization dynamics and also to design novel devices. ${ }^{5-7}$ However, it also faces some problems, such as Joule heating due to Ohmic losses. ${ }^{8,9}$ Recently, micromagnetic simulations have shown that propagating spin waves (SWs) in a ferromagnetic nanowire are able to assist magnetic DW motion. ${ }^{10,11}$ Although many research groups have intensively studied the basic characteristics and the possible applications of SWs in a magnetic nanowire theoretically ${ }^{12-14}$ and experimentally, ${ }^{15-17}$ spin-wave-induced DW motion is a new concept to manipulate domain walls, which could serve, for instance, in fast nonvolatile memory devices due to the high velocity of the propagating SWs. ${ }^{17}$ Additionally, SWs exhibit wave properties, such as superposition and phase shift, which might be useful for logic devices. ${ }^{18}$ Although first micromagnetic simulations have shown that SWs can help to move a DW, ${ }^{10,11}$ they were carried out for ideal wire geometries with no DW pinning. In real systems, the DW can be trapped by edge roughness, grain boundaries, and other pinning sites. Such pinning effects strongly influence the potential landscape for the DW and, consequently, its dynamics. ${ }^{19,20}$ Therefore, in order to simulate the behavior in a more realistic system, it is necessary to investigate the interaction between propagating SWs and a pinned DW. Moreover, the basic mechanisms to move a DW by propagating SWs are unclear. Here, we study the SW-assisted domain wall depinning, which has not been studied so far and it provides useful information about the underlying mechanisms of the interaction between SWs and DWs.

In this paper, we investigate the interaction between propagating SWs and a transverse wall (TW) in a magnetic nanowire. We calculate the TW velocity as a function of the SW frequency. A significant negative TW motion (against the spin-wave propagation direction) due to the angular momentum transfer from the propagating magnons to the TW is observed for the lower frequency range. For high frequencies, three peaks with positive velocities are observed. In order to understand the underlying physics, we calculate the depinning fields and the depinning processes for a TW pinned at a square notch. We find that there are two major mechanisms that contribute to the depinning and motion of the TW. One is the momentum transfer from SW to TW due to the strong SW reflections for the overall SW frequency range. The other mechanism is the collective TW vibration mediated by the internal modes of TW at certain SW frequencies.

\section{MATERIALS, GEOMETRY, AND NUMERICAL MODEL}

Figure 1 shows the simulated structure and the initial spin configuration in our simulations with the corresponding coordinate system. The nanowire is 4000-nm long, 150-nm wide, 5-nm thick, and a head-to-head TW is nucleated at the center of the nanowire and pinned by a square notch $\left(5 \times 5 \mathrm{~nm}^{2}\right)$. We select a cell size of $5 \times 5 \times 5 \mathrm{~nm}^{3}$ and standard material parameter values for permalloy are used in the simulations: saturation magnetization $M_{S}=8.0 \times 10^{5} \mathrm{~A} / \mathrm{m}$, exchange stiffness $A=1.3 \times 10^{-11} \mathrm{~J} / \mathrm{m}$, and a damping constant $\alpha=0.01$. We use the object-oriented micromagnetic framework (OOMMF) ${ }^{21}$ code to solve the Landau-Lifshitz (LL) equation:

$$
\frac{\partial \mathbf{M}}{\partial t}=-\gamma \mathbf{M} \times \mathbf{H}_{\mathrm{eff}}+\frac{\alpha \gamma}{M_{S}} \mathbf{M} \times\left(\mathbf{M} \times \mathbf{H}_{\mathrm{eff}}\right),
$$




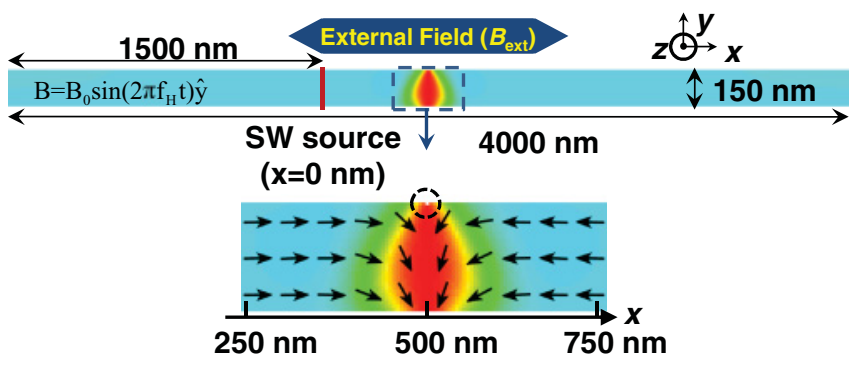

FIG. 1. (Color online) The initial spin configuration of the trapped head-to-head TW nucleated at the position of a square notch in the center of the nanowire. The square notch size is $5 \times 5 \mathrm{~nm}^{2}$. In the bottom we zoom into the central part of the wire to show the internal structure of the TW pinned at the notch. In order to generate SWs, we apply a localized sinusoidal field at a position denoted as the SW source. The SW source is located at $500 \mathrm{~nm}$ from the center of the nanowire. To depin the TW, we apply an external field $B_{\text {ext }}$ along the $\pm x$ direction.

where $\mathbf{M}$ is the vector of local magnetization and $\gamma$ is the gyromagnetic ratio $\left(2.21 \times 10^{5} \mathrm{~m} / \mathrm{As}\right)$. $\mathbf{H}_{\text {eff }}$ is the effective field, which is composed of exchange, anisotropy, magnetostatic, and external field. The magnetocrystalline anisotropy is not considered in this simulation. In order to generate monochromatic SWs, we apply a single harmonic sinusoidal external field $B_{0} \sin \left(2 \pi f_{H} t\right) \mathbf{u}_{\mathbf{y}}$ along the $y$ direction only at a localized area $\left(5 \times 150 \times 5 \mathrm{~nm}^{3}\right)$ at $1500 \mathrm{~nm}(x=$ $0 \mathrm{~nm}$ ) from the left edge of the wire, as indicated in Fig. 1. To prevent SW reflections from the ends, we include absorbing boundary conditions based on gradually increasing the damping constant as approaching the edges of the computational region. ${ }^{22}$ In order to promote the DW depinnig, an external field of $\mathbf{B}_{\mathrm{ext}}=B_{\mathrm{ext}} \mathbf{u}_{\mathbf{x}}$ is applied along the positive or negative $x$ direction. For the pinning site, the depinning fields without SWs for both applied field directions are $B_{\text {dep }}= \pm 1.7 \mathrm{mT}$. In each case, the depinning field is obtained by calculating the equilibrium position of the wall for a series of increasing applied field $\left(B_{\text {ext }}\right)$ in steps of $0.1 \mathrm{mT}$ until a value is reached for which the domain wall depins from the notch.

\section{RESULTS AND DISCUSSIONS}

First, we study the time evolution of the TW position for SWs of various frequencies $\left(f_{H}=5.25,6.25\right.$, and
$11.25 \mathrm{GHz}$, and a total simulation time $\Delta t=20 \mathrm{~ns}$ ) for a free-defect nanowire. To generate propagating coherent SWs, the localized external field is fixed at $B_{0}=10 \mathrm{mT}$ and the TW is located at $x=500 \mathrm{~nm}$ to the right of the SW source. In Fig. 2(a), for the case of $f_{H}=5.25 \mathrm{GHz}$, the TW moves toward the SW source with a constant slope. On the other hand, it can be observed that TWs move along the $+x$ direction for $f_{H}=6.25$ and $11.25 \mathrm{GHz}$. In order to compute the TW velocity $\left(v_{\mathrm{TW}}\right)$ as driven by the excited SWs, the instantaneous value of the normalized $x$ component of the magnetization $\left(\left\langle m_{x}\right\rangle=\left\langle M_{x}\right\rangle /\left\langle M_{S}\right\rangle\right)$ averaged in the wire volume is calculated, and shown in Fig. 2(b) ( $f_{H}=$ $3-30 \mathrm{GHz}$ and $\Delta f_{H}=0.25 \mathrm{GHz}$ ). We observe a negative velocity (corresponding to the TW moving towards the SW source) for low spin-wave frequencies $\left(4.0 \mathrm{GHz}<f_{H}<\right.$ $5.5 \mathrm{GHz}$ ). The maximum velocity in that direction is found $v_{\mathrm{TW}}=-0.22 \mathrm{~m} / \mathrm{s}$. Above $f_{H}=5.5 \mathrm{GHz}$, the TW velocity is positive with three peaks $\left(f_{H}=6.25,11.25\right.$, and $13.75 \mathrm{GHz}$ with resulting velocities of $v_{\mathrm{TW}}=1.32,0.94$, and $0.52 \mathrm{~m} / \mathrm{s}$, respectively). This negative TW velocity has been explained in Refs. ${ }^{23}$ and ${ }^{24}$ based on a transfer of angular momentum from the propagating magnons to the TW. For the other SW frequencies $\left(f_{H}=6.25\right.$ and $\left.11.25 \mathrm{GHz}\right)$, TWs move linearly far from the SW source due to the different physical origins.

Figure 2(c) shows the spatially resolved fast Fourier transformation (FFT) of $M_{z}\left(f_{H}=5.25,6.25\right.$, and $\left.11.25 \mathrm{GHz}\right)$ on a defect-free nanowire with a single domain state up to $x=$ $1500 \mathrm{~nm}$ from the SW source. The SW amplitude is directly proportional $^{25}$ to the FFT amplitude of $M_{z}$. In order to draw the SW amplitude curves, we first calculate the averaged $M_{z} / M_{s}$ and then the peak amplitude of the FFT is obtained. As shown in Fig. 2(c), the shapes of the SW amplitudes depends on the frequencies of the localized oscillating fields. We highlight that there are optimal frequencies for the SW propagations due to the geometry of nanowire. ${ }^{23}$ For the case of $f_{H}=5.25 \mathrm{GHz}$ (black line), SWs are not able to propagate to the end of the nanowire since there is a forbidden gap of the SW dispersion relation. ${ }^{10}$ On the other hand, for the cases of $f_{H}=6.25 \mathrm{GHz}$ (red line) and $11.25 \mathrm{GHz}$ (blue line), there are long-lived propagating SWs up to $x=1500 \mathrm{~nm}$. However, the SW with $f_{H}=$ $11.25 \mathrm{GHz}$ is able to propagate longer than the case of $f_{H}=$ $6.25 \mathrm{GHz}$. Until now, the TW velocity and the SW amplitudes for various SW frequencies are investigated. However, the mechanisms for the TW motion are not revealed yet.
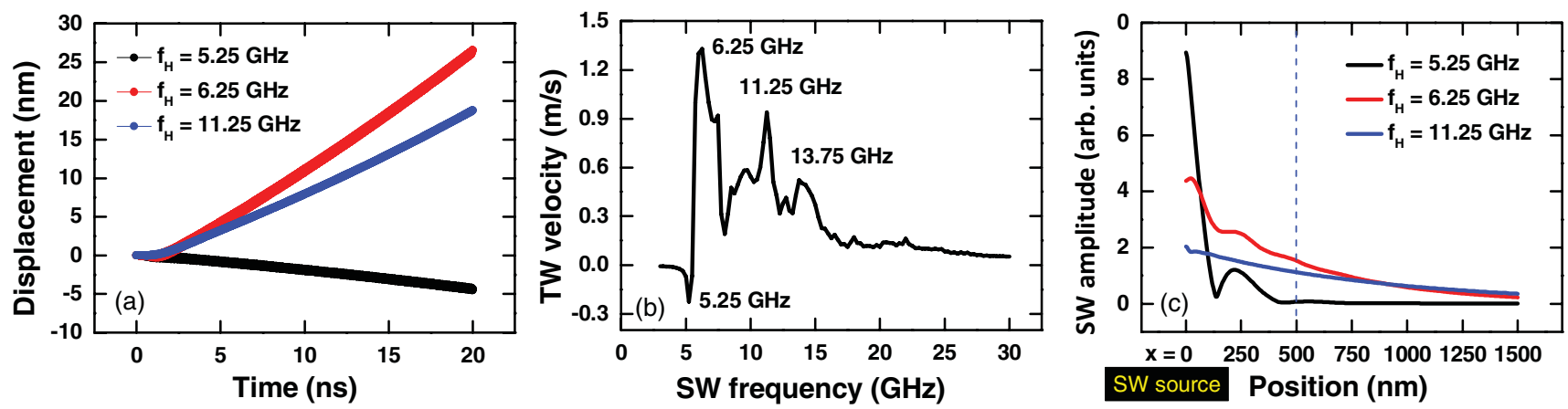

FIG. 2. (Color online) (a) TW displacement as a function of simulation time ( $\Delta t=20 \mathrm{~ns}, f_{H}=5.25,6.25$, and $11.25 \mathrm{GHz}$ ). (b) The TW velocity $\left(v_{\mathrm{TW}}\right)$ as a function of $\mathrm{SW}$ frequency $\left(f_{H}=3 \mathrm{GHz} 30 \mathrm{GHz}, \Delta f_{H}=0.25 \mathrm{GHz}\right.$, and $\Delta t=20 \mathrm{~ns}$ ). (c) The SW amplitude (=FFT amplitudes of $\left.M_{z}\right)$ as a function of the position from the SW source $(x=0)$ without a TW $\left(f_{H}=5.25,6.25\right.$, and $\left.11.25 \mathrm{GHz}\right)$ 

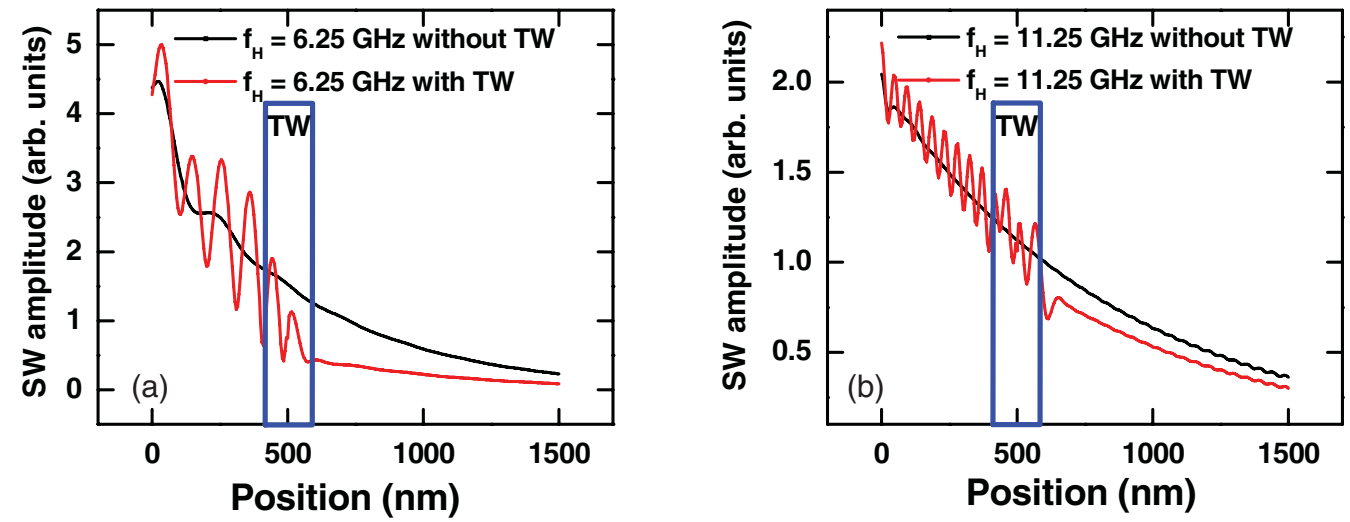

FIG. 3. (Color online) Spin-wave amplitudes with and without a TW for two spin-wave frequencies (a) $f_{H}=6.25 \mathrm{GHz}$ and (b) $f_{H}=$ 11.25 GHz. These frequencies correspond to the positive peaks in Fig. 2(b).

In order to understand the underlying physics about the TW motion due to the propagating SWs, we calculate the SW amplitudes as a function of the $x$ position with and without a TW for $f_{H}=6.25 \mathrm{GHz}$ and $11.25 \mathrm{GHz}$. These two frequencies correspond to the positive peaks of the TW velocity [see Fig. 2(b)]. In Fig. 3, black lines and red lines indicate the SW amplitudes without and with a TW, respectively. To hold the TW at the equilibrium position, we introduce a small rectangular notch $(5 \mathrm{~nm} \times 5 \mathrm{~nm})$ at the center of the nanowire as shown in Fig. 1. First, we highlight that there are standing waves due to the SW reflection for both frequencies. For $f_{H}=$ $6.25 \mathrm{GHz}$, we observe that SWs are not able to propagate across the TW and most of the SW is reflected at the TW position $(x=500 \mathrm{~nm})$ in Fig. 3(a). In this case, the SW reflection is the dominant mechanism for the TW motion by the propagating SWs. However, for $f_{H}=11.25 \mathrm{GHz}, \mathrm{SWs}$ can penetrate
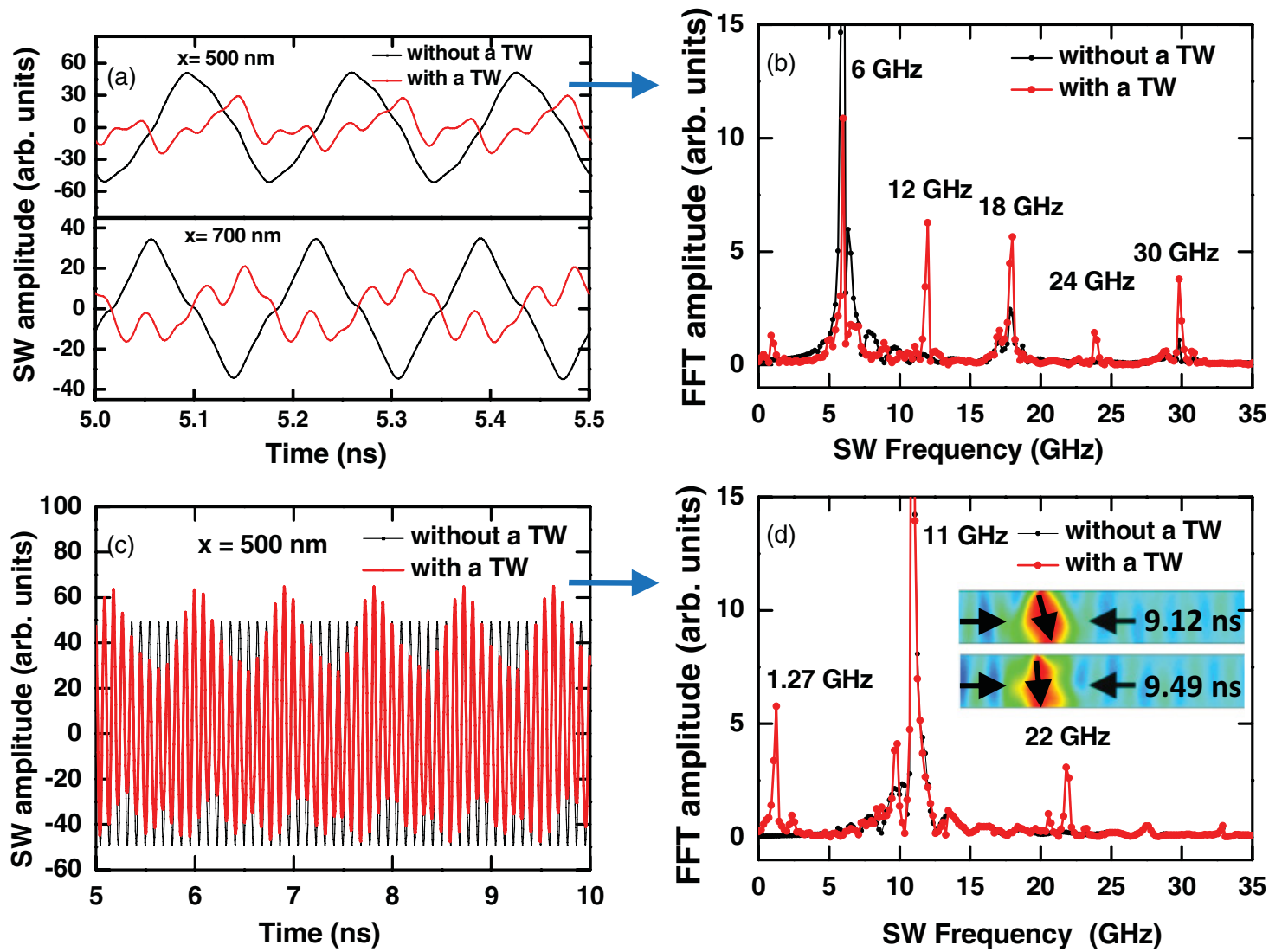

FIG. 4. (Color online) (a) The SW amplitudes as a function of time ( $t=5.0-5.5 \mathrm{~ns}$ with $f_{H}=6.0 \mathrm{GHz}, B_{0}=200 \mathrm{mT}$, and $B_{\text {ext }}=0.5 \mathrm{mT}$ ) at the TW position $(x=500 \mathrm{~nm}$ and $x=700 \mathrm{~nm}$ ) (black line, the SW amplitude without a TW; red line, the SW amplitude with a TW). (b) The FFT spectra of the SW amplitude for the case of (a) at the TW position $(x=500 \mathrm{~nm})$. (c) The SW amplitudes as a function of time at $x=$ $500 \mathrm{~nm}\left(t=5.0-10.0 \mathrm{~ns}\right.$ with $f_{H}=11.0 \mathrm{GHz}, B_{0}=200 \mathrm{mT}$, and $\left.B_{\text {ext }}=0.8 \mathrm{mT}\right)$. (d) The FFT spectrum of the SW amplitude for the case of (c) at the TW position. Inset shows the snapshots of the $y$-component magnetization for two different simulation times. 

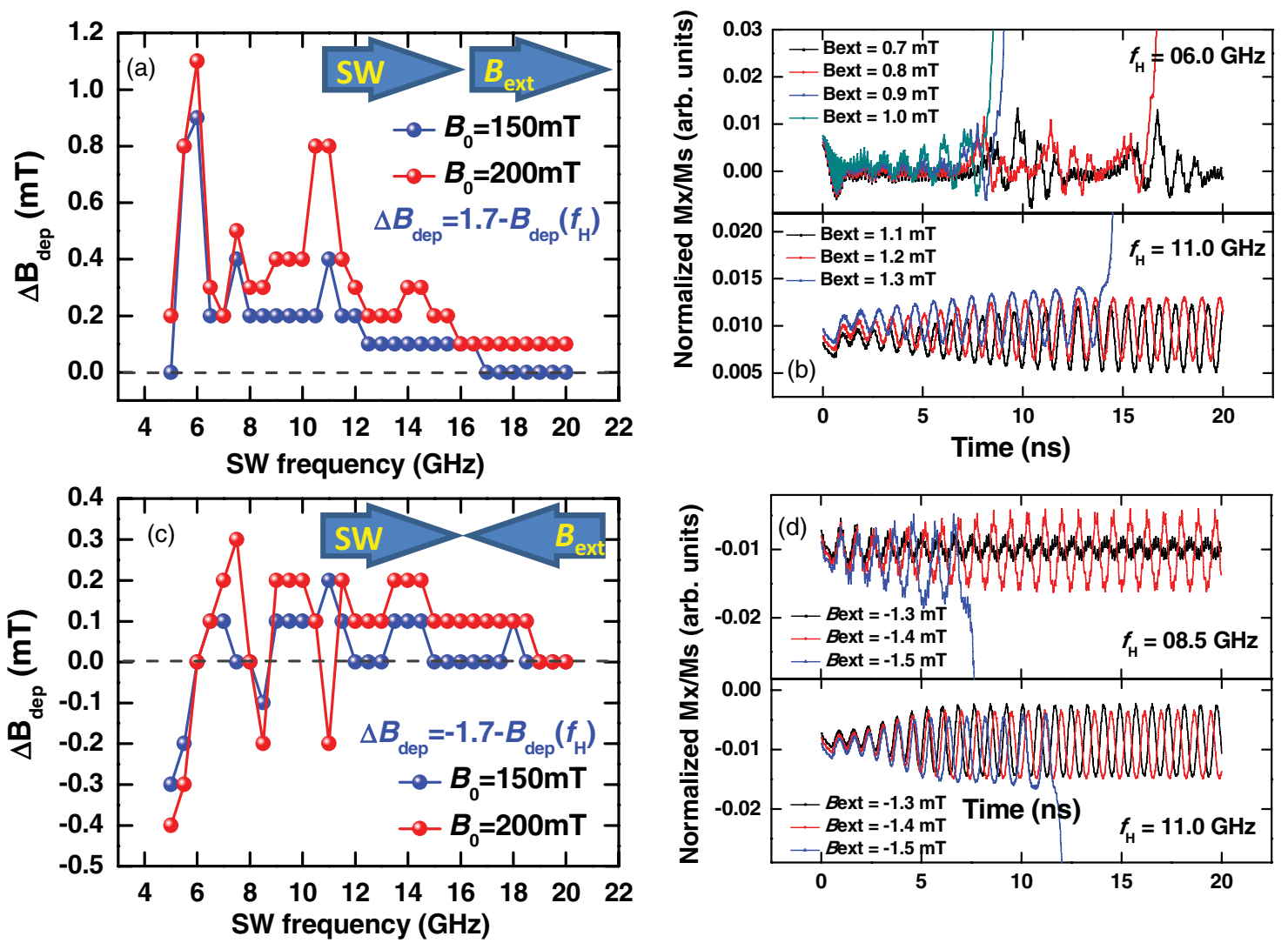

FIG. 5. (Color online) (a and c) $\Delta B_{\text {dep }}$ calculations with the external fields along the $\pm x$ direction $\left(B_{0}=150\right.$ and $200 \mathrm{mT}$ ). (a) $\Delta B_{\text {dep }}$ calculations with the external fields along the $+x$ direction $\left[\Delta B_{\mathrm{dep}} \equiv 1.7 \mathrm{mT}-B_{\mathrm{dep}}\left(f_{H}\right)\right]$. (b) The normalized $M_{x} / M_{S}$ curves with various external fields (up, $f_{H}=6.0 \mathrm{GHz}$; down, $f_{H}=11.0 \mathrm{GHz}$ ). $B_{0}=150 \mathrm{mT}$ is used (blue line, $f_{H}=6.0 \mathrm{GHz}$ and $B_{\mathrm{dep}}=1.0 \mathrm{mT}$; red line, $f_{H}=$ $11.0 \mathrm{GHz}$ and $B_{\mathrm{dep}}=1.3 \mathrm{mT}$ ). (c) $\Delta B_{\text {dep }}$ calculations with the external fields along the $-x$ direction $\left[\Delta B_{\text {dep }} \equiv-1.7 \mathrm{mT}-B_{\text {dep }}\left(f_{H}\right)\right]$. (d) The normalized $M_{x} / M_{S}$ curves with various external fields (up, $f_{H}=8.5 \mathrm{GHz}$; down, $f_{H}=11.0 \mathrm{GHz}$ ). $B_{0}=200 \mathrm{mT}$ is used.

the TW with relatively small reflection. Even though TWs move with relatively high velocities at $f_{H}=6.25 \mathrm{GHz}$ and 11.25 GHz as shown in Fig. 2(a), this implies that the details of the TW propagation mechanisms are totally different. On the contrary, Han et al. ${ }^{10}$ pointed only to one physical origin of the SW-induced TW motion. They claimed that a TW moves resonantly at specific SW frequencies. However, here we show that there is a second mechanism, which we discuss in the present study.

In order to investigate further these two different mechanisms, we calculate the propagating $\mathrm{SW}$ amplitudes as a function of time $(t=5.0-5.5 \mathrm{~ns})$ for the pinned TW. The SW amplitudes at the center of the TW $(x=500 \mathrm{~nm})$ and to the right side of the TW $(x=700 \mathrm{~nm})$ with $B_{0}=200 \mathrm{mT}$ for two different frequencies $\left(f_{H}=6\right.$ and $\left.11 \mathrm{GHz}\right)$ are depicted in Figs. 4(a) and 4(c), respectively. In order to take a closer look at the depinning mechanisms with the propagating SW, we apply external fields, $0.1 \mathrm{mT}$ smaller than the depinning fields ( $B_{\text {ext }}=0.5$ and $0.8 \mathrm{mT}$ for $f_{H}=6$ and $11 \mathrm{GHz}$, respectively). We observe that the SW amplitude already decreases from 50 (at $x=500 \mathrm{~nm}$ ) to 30 (at $x=700 \mathrm{~nm}$ ) without a TW in Fig. 4(a) (black lines top and bottom figures). When we introduce a TW, the SW profiles strongly deviate from the simple sinusoidal function, which is clearly shown in the SW amplitude at $x=500 \mathrm{~nm}$ in Fig. 4(a). Figure 4(b) shows the
FFT of the SW amplitude at $x=500 \mathrm{~nm}$ for both cases with and without a TW in Fig. 4(a). Higher order harmonics ( $f=$ $12,18,24$, and $30 \mathrm{GHz}$ ) of the main frequency $f_{H}=6 \mathrm{GHz}$ are excited as can be observed in Fig. 4(b). Without a TW, there are higher order harmonics of odd number $(f=18,30 \mathrm{GHz})$, but they are very small, and these higher order harmonics stem from the fact that the wave front of the $\mathrm{SW}$ at $f_{H}=6 \mathrm{GHz}$ is not sinusoidal [see Fig. 4(a)].

Figure 4(c) shows the SW amplitudes for $f_{H}=11 \mathrm{GHz}$ as a function of time $(t=5.0-10.0 \mathrm{~ns})$. As mentioned before, the decrease of the SW amplitude due to the existence of TW is smaller than the $f_{H}=6 \mathrm{GHz}$ [see Fig. 4(a)]. One major difference is the beating of the SW amplitudes at $x=500 \mathrm{~nm}$. The amplitudes of SW change with a frequency of $1.27 \mathrm{GHz}$. The clear peak of $1.27 \mathrm{GHz}$ is seen in Fig. 4(d) together with $11-$ and $22-\mathrm{GHz}$ peaks. The origin of the 1.27-GHz peak is the vibration or collective oscillation of the whole TW structure. ${ }^{10}$ At $f_{H}=11 \mathrm{GHz}$, SWs generate such a collective motion. The internal mode can be excited with the corresponding propagating SW, but the absorbed energy transfers to the collective motion of the whole TW. Although, a significant FFT amplitude is present at higher harmonic $\mathrm{SW}$ frequency $\left(f_{H}=22 \mathrm{GHz}\right)$ due to the $\mathrm{SW}$ reflection, the amplitude is relatively smaller than the case of $f_{H}=6 \mathrm{GHz}$. The inset shows the $y$ components of the magnetization for 
two different simulations times $(t=9.12 \mathrm{~ns}$ and $9.49 \mathrm{~ns}$, respectively) with $f_{H}=11 \mathrm{GHz}$. The whole TW is resonantly excited. Note that the vibrations are not symmetric due to the external field.

In order to reveal more details of the differences between $f_{H}=6$ and $11 \mathrm{GHz}$, we calculate the TW dynamics and the depinning fields as a function of SW frequency. Figures 5(a) and $5(\mathrm{c})$ show the depinning field differences $\left[\Delta B_{\text {dep }}\left(f_{H}\right)\right]$ for positive and negative $B_{\text {ext }}$ along the $x$ direction. For both cases, the depinning fields without SWs are $\pm 1.7 \mathrm{mT}$. We calculate $\Delta B_{\text {dep }}$ as a function of SW frequency $\left(f_{H}<\right.$ $20 \mathrm{GHz}$ and $\Delta f_{H}=0.5 \mathrm{GHz}$ ) for $B_{0}=150$ and $200 \mathrm{mT}$. To prevent TW oscillations when the external field is changed, we calculate the equilibrium spin configurations for each applied field before applying the localized excitation that generates SWs. In Fig. 5(a), both momentum transfer and the collective motion of the TW assist the depinning of TW due to the fact that the directions of the propagating SW and the external field are parallel. Although the SW amplitudes decrease when the SW frequency increases, we observe four positive peaks of $\Delta B_{\text {dep }}\left(\equiv 1.7-B_{\text {dep }}\left(f_{H}\right)\right)$ at $f_{H}=6.0,7.5,10.5-11.0$, and $13.5-14.0 \mathrm{GHz}$ in Fig. 4(a). The normalized $M_{x} / M_{S}$ as a function of time for both $f_{H}=6$ and $11 \mathrm{GHz}$ with $B_{0}=$ $150 \mathrm{mT}$ as shown in Fig. 5(b) is calculated. At $f_{H}=6 \mathrm{GHz}$, the TW interacts with the propagating SW and then depins $\left(B_{\text {ext }}=0.8-1.0 \mathrm{mT}\right)$ or fluctuates $\left(B_{\text {ext }}=0.7 \mathrm{mT}\right)$. For $f_{H}=6 \mathrm{GHz}$, the collective motion of TW is not observed, except for $B_{\text {ext }}=1.0 \mathrm{mT}$ as shown in the top panel of Fig. 5(b). Only small harmonic oscillations are found for $B_{\text {ext }}$ $=1.0 \mathrm{mT}$ as we already explained in Figs. 4(a) and 4(b). In addition, for the second peak $\left(f_{H}=7.5 \mathrm{GHz}\right)$, the TW fluctuates or directly depins without harmonic oscillations (not shown here). However, at $f_{H}=11 \mathrm{GHz}$, the propagating SWs generate the collective oscillation of the TW for all external fields ( $B_{\text {ext }}=1.1-1.3 \mathrm{mT}$ ) as shown in the bottom panel of Fig. 5(b). It should be mentioned that the intrinsic characteristic TW oscillation frequencies are about 1.25, 1.15, and $0.95 \mathrm{GHz}$ with $B_{\mathrm{ext}}=1.1,1.2$, and $1.3 \mathrm{mT}$, respectively.

Figure 5(c) shows $\Delta B_{\text {dep }}$ with the negative external field along the $-x$ direction $\left(\equiv-1.7-B_{\text {dep }}\left(f_{H}\right)\right)$. In this case, the role of the SWs is even clearer than for the positive external field case. For $f_{H}=5.0-5.5 \mathrm{GHz}$, the $\mathrm{SW}$ decays very quickly and exists only in the left side of the TW as we discussed earlier [see Fig. 2(c)]. Therefore the TW is attracted to the SW source, and it helps the depinning processes to the $-x$ direction with the negative external field. Thus, a negative $B_{\text {dep }}$ is obtained at that frequency range as shown in Fig. 5(c). For $f_{H}>6.0 \mathrm{GHz}$, $\Delta B_{\text {dep }}$ is always positive except 8.5 and $11.0 \mathrm{GHz}$ for $B_{0}=$ $200 \mathrm{mT}$. The positive $\Delta B_{\text {dep }}$ implies the SW prevents the depinning processes against the negative external field. This is consistent with the fact that the propagating SW moves a TW in the SW propagating direction. However, there are negative $\Delta B_{\text {dep }}$ at specific frequencies, $f_{H}=8.5$ and $11.0 \mathrm{GHz}$. At the frequencies (internal modes), the SW helps the depinning processes in spite of the opposite SW propagation direction to the external magnetic field. This is further clear evidence of the different mechanisms dominating at $f_{H}=6$ and $11 \mathrm{GHz}$, respectively. At the specific frequencies, we plot the normalized $M_{x} / M_{S}$ as a function of the time in Fig. 5(d) with various negative external fields. The top and bottom panels $\left(f_{H}=8.5\right.$ and $11.0 \mathrm{GHz}$ ) show clear oscillations of the TW, the collective oscillation of the TW. The oscillation frequencies are around $1 \mathrm{GHz}$, as already explained in Fig. 4(d). Although the small peak at $f_{H}=7.5 \mathrm{GHz}$ in Fig. 5(a) is observed, it is unclear whether the frequency is a new internal mode or not since the propagating SWs and the applied field have the same directions. However, we observe a clear new internal mode for $f_{H}=8.5 \mathrm{GHz}$ in Fig. 5(c), and it is the external fields that are able to give rise to new internal modes.

\section{CONCLUSIONS}

In conclusion, we have numerically investigated the interactions between the propagating SWs and a TW in a magnetic nanowire. First, we have calculated the TW velocity with propagating SWs as a function of the excited SW frequency in a defect-free nanowire. At $f_{H}=5.25 \mathrm{GHz}$, negative TW motion was observed since a transfer of angular momentum from the propagating magnons to the TW. Above $f_{H}=5.5 \mathrm{GHz}$, we have observed three positive velocity peaks $\left(f_{H}=6.25,11.25\right.$, and $13.75 \mathrm{GHz}$ ). We have found from the calculations of SW amplitudes and the depinning fields that there are two different mechanisms to excite and depin the TW. One is the momentum transfer from SWs to the TW-accompanied strong SW reflections at $f_{H}=6 \mathrm{GHz}$. The other mechanism is the collective TW oscillation mediated by the internal modes of TW at certain SW frequencies $\left(f_{H}=8.5\right.$ and $\left.11 \mathrm{GHz}\right)$. Especially, a new internal mode is found for the uniform external field at $f_{H}=8.5 \mathrm{GHz}$. Based on our study, we can conclude that the details of the interaction between propagating SWs and TWs depends on not only the propagating SW frequencies, but also the collective TW vibrations of the spin structure in the nanowires.

\section{ACKNOWLEDGMENTS}

This work is supported by the European Union Research Training Network SPINSWITCH (Contract No. MRTN-CT2006-035327), the Samsung Advanced Institute of Technology, the DFG (SFB 767; Grant No. KL 1811), the Swiss National Science Foundation, the European Research Council via a Starting Independent Researcher Grant (Grant No. ERC2007-Stg 208162), the Spanish Government under Project No. MAT2011-28532-C03-01, and the National Research Foundation of Korea grants funded by the Ministry of Education, Science, and Technology (Grants No. 2010-0023798 and No. 2010-0022040).

\footnotetext{
*klaeui@uni-mainz.de; Current address: Institut für Physik, Universität Mainz, 55128 Mainz, Germany.

†cyyou@inha.ac.kr
}

${ }^{1}$ D. A. Allwood, G. Xiong, C. C. Faulkner, D. Alkinson, D. Petit, and R. P. Cowburn, Science 309, 1688 (2005).

${ }^{2}$ S. S. P. Parkin, US Patent No. 6834005 (2004). 
${ }^{3}$ C.-Y. You, Appl. Phys. Lett. 92, 192514 (2006).

${ }^{4}$ D. Ilgaz, M. Kläui, L. Heyne, F. Zinser, S. Krzyk, M. Fonin,

U. Rüdiger, D. Backes, and L. J. Heyderman, Appl. Phys. Lett. 93, 132503 (2008).

${ }^{5}$ A. Yamaguchi, T. Ono, S. Nasu, K. Miyake, K. Mibu, and T. Shinjo, Phys. Rev. Lett. 92, 077205 (2004).

${ }^{6}$ M. Kläui, P.-O. Jubert, R. Allenspach, A. Bischof, J. A. C. Bland, G. Faini, U. Rüdiger, C. A. F. Vaz, L. Vila, and C. Vouille, Phys. Rev. Lett. 95, 026601 (2005).

${ }^{7}$ J. Grollier, P. Boulenc, V. Cros, A. Hanzić, A. Vaurés, and A. Fert, Appl. Phys. Lett. 83, 509 (2003).

${ }^{8}$ C.-Y. You, I. M. Sung, and B.-K. Joe, Appl. Phys. Lett. 89, 222513 (2006).

${ }^{9}$ C.-Y. You and S.-S. Ha, Appl. Phys. Lett. 91, 022507 (2007).

${ }^{10}$ D.-S. Han, S.-K. Kim, J.-Y. Lee, S. J. Hermsoerfer, H. Schutheiss, B. Leven, and B. Hillebrands, Appl. Phys. Lett. 94, 112502 (2009).

${ }^{11}$ M. Jamali, H. Yang, and K.-J. Lee, Appl. Phys. Lett. 96, 242501 (2010).

${ }^{12}$ K. Y. Guslienko, R. W. Chantrell, and A. N. Slavin, Phys. Rev. B 68, 024422 (2003).

${ }^{13}$ K.-S. Lee, D.-S. Han, and S.-K. Kim, Phys. Rev. Lett. 102, 127202 (2009).

${ }^{14}$ S.-M. Seo, K.-J. Lee, H. Yang, and T. Ono, Phys. Rev. Lett. 102, 147202 (2009).
${ }^{15}$ M. Covington, T. M. Crawford, and G. J. Parker, Phys. Rev. Lett. 89, 237202 (2002).

${ }^{16}$ V. Vlaminck and M. Bailleul, Science 322, 410 (2008).

${ }^{17}$ K. Sekiguchi, K. Yamada, S. M. Seo, K. J. Lee, D. Chiba, K. Kobayashi, and T. Ono, Appl. Phys. Lett. 97, 022508 (2010).

${ }^{18}$ R. Hertel, W. Wulfhekel, and J. Kirschner, Phys. Rev. Lett. 93, 257202 (2004)

${ }^{19}$ L. Heyne, M. Kläui, D. Backes, P. Möhrke, T. A. Moore, J. G. Kimling, O. Boulle, U. Rüdiger, L. J. Heyderman, A. Fraile Rodríguez, F. Nolting, K. Kirsch, and R. Mattheis, J. Appl. Phys. 103, 07D928 (2008).

${ }^{20}$ J.-S. Kim, O. Boulle, S. Verstoep, L. Heyne, J. Rhensius, M. Kläui, L. J. Heyderman, F. Kronast, R. Mattheis, C. Ulysse, and G. Faini, Phys. Rev. B 82, 104427 (2010).

${ }^{21}$ http://math.nist.org/oommf/.

${ }^{22}$ G. Consolo, L. Lopez-Diaz, L. Torres, and B. Azzerboni, IEEE Trans. Magn. 43, 2974 (2007); Phys. Rev. B 75, 214428 (2007).

${ }^{23}$ P. Yan, X. S. Wang, and X. R. Wang, Phys. Rev. Lett. 107, 177207 (2011).

${ }^{24}$ A. V. Mikhailov and A. I. Yaremchuk, JETP Lett. 39, 354 (1984).

${ }^{25}$ S.-M. Seo, H.-W. Lee, H. Kohno, and K.-J. Lee, Appl. Phys. Lett. 98, 012514 (2011). 